\title{
DISEÑO DE UN MODELO BASADO EN AGENTES PARA SIMULAR EL CRECIMIENTO URBANO EN EL CORREDOR DEL HENARES (COMUNIDAD DE MADRID)
}

\author{
Carolina Cantergiani \\ Montserrat Gómez Delgado \\ Departamento de Geología, Geografía y Medio Ambiente. Universidad de Alcalá \\ carolina.carvalho@uah.es, montserrat.gomez@uah.es
}

\section{RESUMEN}

Ante el impacto que la expansión urbana ejerce sobre el planeta y sobre su población, resulta esencial la utilización de técnicas de simulación que nos permitan elaborar escenarios sobre la evolución de estos sistemas. En este sentido, los Modelos Basados en Agentes (MBA) se presentan como una alternativa prometedora. Los MBA son modelos celulares y dinámicos que se basan en reglas de decisión y que permiten incorporar el comportamiento de los agentes involucrados en un determinado sistema. En esta investigación proponemos un diseño de un MBA para simular el crecimiento urbano en el Corredor del Henares (Comunidad de Madrid, España), teniendo en cuenta su dinámica urbana, los principales agentes involucrados en este proceso de expansión, su aspecto comportamental, así como la información espacial disponible. El principal objetivo es disponer de un diseño conceptual sólido, para posteriormente demostrar que los MBA pueden ser útiles como herramienta alternativa para simular el crecimiento urbano a escala subregional.

Palabras clave: modelos basados en agentes, simulación de crecimiento urbano, Corredor del Henares, Comunidad de Madrid (España), Sistemas de Información Geográfica.

Fecha de recepción: marzo 2014.

Fecha de aceptación: noviembre 2014. 


\section{ABSTRACT}

Due to urban sprawl impact over the planet and the population, the use of simulation techniques that generate scenarios regarding the evolution of those systems is imperative. Agent-based models (ABM) are presented as a promising alternative. ABM is a dynamic cellular model based on decision rules guided by the behaviour of agents. In the present work, we propose the design of an ABM to simulate urban growth in 'Corredor del Henares' (Community of Madrid, Spain), considering its urban dynamics and the main involved agents, as well as their particular behaviour and the available data. The main goal is to have a solid conceptual design in order to prove, in a short time, that ABM can be an alternative tool to simulate urban growth at a sub-regional scale.

Keywords: agent-based models, urban growth simulation, 'Corredor del Henares', Community of Madrid (Spain), Geographic Information Systems.

\section{INTRODUCCIÓN}

El impacto que la expansión urbana está y seguirá ocasionando sobre el planeta y sobre la población que lo habita es innegable. Ante esta situación, resulta esencial la utilización de técnicas de simulación que nos permitan elaborar distintos escenarios sobre la evolución de estos sistemas y así planificar de una forma racional y sostenible los futuros desarrollos urbanos. En este sentido, es cada día más habitual la utilización de modelos de simulación por parte de científicos expertos en materia territorial. Estos modelos, enfocados a la simulación de escenarios futuros (Paegelow y Camacho Olmedo, 2008; Carsjens, 2009; Hansen, 2010; Santé et al., 2010) se presentan como potentes herramientas para analizar y evaluar cuestiones ambientales y territoriales y, en general, cuestiones relacionadas con los procesos de toma de decisiones en planificación, contexto en el que se integra el presente trabajo.

En general, estos modelos utilizados en simulaciones aplicadas a las ciencias sociales suelen reproducir diferentes tipos de análisis y escenarios prospectivos, que pueden ser desagrupados en predictivos (carácter prescriptivo), exploratorios (carácter explicativo), o, en algunos casos, normativos (deseables u óptimos) (Parker et al., 2001; Aguilera Benavente et al., 2010). Para atender a los diferentes enfoques de estos tres grupos de escenarios, se pueden utilizar distintos tipos de modelos de simulación según la orientación deseada.

La aplicación de los MBA en el campo de la simulación urbana podría encuadrase en uno o más de los enfoques presentados (exploratorio, predictivo o normativo). Son considerados como un laboratorio en miniatura, donde los atributos, el comportamiento de los agentes y el medio en el cual se establecen pueden ser alterados, y sus repercusiones pueden ser observadas a lo largo de la ejecución de varias simulaciones (Castle y Crooks, 2006). De esta forma, encajarían en los escenarios ¿what if? (exploratorios), por tratarse de modelos que sirven, no solo para extrapolar tendencias, sino también para visualizar las probables modificaciones que se producirían en el territorio a partir de determinados cambios en las variables iniciales. Por otro lado, los MBA también podrían utilizarse en el contexto de escenarios exploratorios estratégicos, por su capacidad para permitir plantear cómo la realidad debería ser, o sería, 
bajo determinadas circunstancias, aunque sin pretender nunca reproducir los sistemas actuales (Parker, Berger y Manson, 2001).

De forma general, si acotamos el objetivo del modelo a los escenarios futuros de carácter exploratorio, y considerando su aplicación a cuestiones urbanas, los modelos más comúnmente empleados en estos casos son los modelos celulares. Aunque estos modelos se introdujeron ya en los años 60, siguen siendo actualmente muy utilizados y su evolución a lo largo de estos años ha generado una nueva visión de la simulación (Benenson y Torrens, 2004; Batty, 2005) que se ha traducido en el desarrollo de una multitud de herramientas para este fin (White et al., 1997; López et al., 2001; Gómez Delgado y Barredo Cano, 2005; Paegelow y Camacho Olmedo, 2008; Aguilera Benavente et al., 2010; Plata Rocha et al., 2011). Quizás los modelos celulares más conocido en las ciencias sociales, por su amplia aplicación a estudios espaciales, sean los modelos basados en Autómatas Celulares (AC).

De forma general, estos modelos basados en AC funcionan de forma que, a partir de un conjunto de celdas, con un estado determinado, unas reglas de transición y en función de su comportamiento respecto a la vecindad inmediata, cada unidad se comporta dinámicamente cambiando su estado inicial y conformando patrones espaciales. Entre otros muchos documentos, podemos encontrar una compilación de numerosos trabajos de AC en los anales del Congreso Cellular Automata Modeling for Urban and Spatial Systems (Norte Pinto et al., 2012).

Los AC son, en cierta forma, el fundamento de otro tipo de modelo celular, los Modelos Basados en Agentes, que también presentan un elevado potencial de simulación urbana y alta capacidad de modelación. Los MBA se han visto influenciados de forma significativa, no sólo por el desarrollo de la teoría de los autómatas celulares (en la física y matemática), sino también por la ciencia de la computación (inteligencia artificial distribuida y tecnología de agentes) (Gilbert y Troitzsch, 2005).

Aunque tanto los AC como los MBA partan de la misma estructura (celular), se basen en reglas de decisión y sean modelos altamente dinámicos, los MBA están poco a poco ocupando un vacío no cubierto por los AC, al agregar agentes en su configuración. A través de estos agentes, los MBA incorporan un aspecto comportamental, ya que éstos deben representar los principales elementos involucrados en el fenómeno estudiado y, a su vez, son los actores responsables de promover los cambios que se pretenden simular. Las características de algunos de los agentes como la intuición, el aprendizaje o la deducción, no se consideran en los mencionados AC (Cantergiani, 2011a).

De manera resumida podemos decir que los MBA son modelos celulares que simulan un determinado fenómeno a través del comportamiento de agentes autónomos que actúan teniendo en cuenta su estado y características propias, la percepción de su entorno, así como sus propios intereses, reflejados en una serie de reglas de decisión que les permiten a alcanzar un objetivo concreto, provocando los consecuentes cambios en la estructura inicial del sistema.

De esta forma, considerando las reacciones de los agentes ante un determinado fenómeno, los MBA permiten a los investigadores estudiar cómo el comportamiento del sistema emerge y está conectado a las características y comportamiento de sus componentes individuales, o entender cómo el propio sistema afecta al individuo (Railsback y Grimm, 2009). 
El presente trabajo se centra en la aportación de los MBA a la exploración del fenómeno de crecimiento urbano a escala subregional y al mejor entendimiento de esta dinámica, con el objetivo de apoyar y mejorar las tareas de planificación. La Comunidad de Madrid (CM), donde se sitúa el área de estudio, presenta una intensa dinámica urbana en los últimos años que es importante comprender, y que hace más necesario, si cabe, la planificación del territorio que todavía queda por urbanizar, teniendo en cuenta, además, que se trata de una región con un volumen importante de territorio bajo diferentes figuras de protección.

Como hemos mencionado, los modelos celulares son idóneos para realizar análisis espaciales con un enfoque urbano, especialmente en la simulación de escenarios futuros. Sin embargo, la simulación urbana en España en general, y en la CM en particular, no está suficientemente explorada y menos si consideramos la utilización de MBA. Aun así, podemos encontrar algunos estudios que se centran en explicar la expansión urbana de la CM, en identificar sus principales factores o en definir patrones de crecimiento. Algunos de ellos proponen proyectar posibles desarrollos futuros a través de la modelización urbana utilizando distintas metodologías. Por ejemplo, Barredo Cano y Gómez Delgado (2008) proponen tres escenarios urbanos futuros basados en el Panel Intergubernamental sobre el Cambio Climático (Intergovernmental Panel on Climate Change - IPCC) aplicados a la región de Madrid, simulados a partir de un modelo basado en AC. Otro ejemplo son los resultados del proyecto europeo TiGrESS (Encinas et al., 2006) que muestran un modelo conceptual de cambios de usos del suelo en la CM. En el proyecto SIMURBAN, se aplican técnicas de evaluación multicriterio y modelos basados en autómatas celulares para simular tres escenarios diferentes de crecimiento urbano de la Comunidad de Madrid (Plata Rocha et al., 2010; Plata Rocha, Gómez Delgado y Bosque Sendra, 2011). Por su parte, Gallardo y Martínez Vega (2012) aplican el modelo CLUE e IDRISI Land Change Modeller para estudiar y proyectar cambios en el mismo área de estudio. Asimismo, en el trabajo reciente de Van Delden et al. (2012) se emplean los AC con el modelo METRONÁMICA de RIKS (Research Institute for Knowledge Systems), para simular la expansión urbana regional. Estos son algunos ejemplos de aplicaciones afines a la del presente documento, y que además se aplican al mismo ámbito geográfico. Sin embargo, se desconoce la existencia de aplicación específica de modelos basados en agentes para simulación urbana en España.

Con vistas a cubrir este vacío, presentamos el diseño de un MBA propuesto para simular el crecimiento urbano en una parte de la CM, cuyo trabajo se enmarca en un contexto más amplio que intenta mostrar la utilidad de los MBA como herramienta alternativa para simular este crecimiento urbano a escala subregional, incluso presentando algunas ventajas sobre los AC.

\section{LOS MODELOS BASADOS EN AGENTES APLICADOS A ESTUDIOS URBANOS}

Los MBA, atendiendo a su estructura, están integrados por un conjunto de agentes o entidades, parcial o totalmente autónomas, que actúan de manera racional y reactiva de acuerdo con sus percepciones del exterior y el estado de su conocimiento, relacionándose entre sí y con su medio, con la intención de alcanzar un objetivo concreto.

Hasta el momento, los MBA han sido ampliamente utilizados en distintos campos como la ecología, la informática, la biología, la economía o las ciencias políticas o sociales (es 
posible encontrar un número importante de ejemplos en Grimm et al. (2006)). En el ámbito de aplicación de MBA a las ciencias sociales, destacan dos de los primeros modelos desarrollados: el 'Modelo de segregación', de Thomas Shelling (1971) y el modelo 'Sugarscape', de Epstein y Axtell (1996). Sin embargo, estos modelos todavía no han sido suficientemente explorados en su aplicación a la simulación de fenómenos explícitamente espaciales como el crecimiento urbano.

Entre las aplicaciones urbanas de MBA, encontramos algunos ejemplos que se han centrado en analizar elementos a escala local considerando los individuos como agentes. En este sentido, hallamos trabajos que simulan los movimientos de coches y peatones en el tráfico urbano (Jiang, 2000; Torrens, 2012), dinámicas de evacuación de emergencia en ambientes cerrados teniendo en cuenta rutas, tiempo, condiciones de los agentes, etc. (Castle, 2007), modelos de segregación social (Benenson et al., 2002; Crooks, 2006; Feitosa et al., 2011), modelos de asignación de empleo y residencias (Crooks, 2006), dinámicas económicas de mercado relacionada con los usos del suelo y distribución residencial (Filatova et al., 2009; Ettema, 2011). Otras aplicaciones se detienen más en explicar fenómenos relacionados con la ciudad y la asignación de viviendas a un nivel intraurbano (Dearden y Wilson, 2012; Jordan et al., 2012).

La mayoría de los trabajos de MBA en el ámbito urbano se aplica a escala de individuo, pero dado que tienen funcionalidades que permiten analizar fenómenos complejos, consideramos que podrían aplicarse también a escalas menos detalladas (el histórico de aplicación de los MBA en otros campos de estudio sostiene esta hipótesis), y partimos del supuesto de que también pueden ser útiles para simular el crecimiento urbano a escala subregional.

En algunos casos se han planteado MBA con estos objetivos. Así, en el trabajo de J. Barros (2004) se lleva a cabo una modelización del crecimiento urbano en Latinoamérica estudiando concretamente el fenómeno de periferización en diferentes ciudades. Por otro lado, el modelo SIMPOP (Sanders et al., 1997) es un buen ejemplo de aplicación de dinámicas urbanas a escalas intermedias, que viene siendo desarrollado y perfeccionado hace algunos años. Siguiendo la misma línea de modelización del crecimiento urbano, Benenson y Torrens (2004) presentan en su libro una introducción teórica sobre la aplicabilidad de modelos como los MBA en análisis de la dimensión urbana y publican una serie de artículos relacionados (Torrens, 2006). Algunos otros ejemplos que sirven de referencia se encuentran en el libro de Heppenstall y otros (2012). Asimismo, muchos de los resultados de estos trabajos están todavía en fase de desarrollo, actualización o revisión, dando fe de la etapa inicial en la que se encuentra la modelización basada en agentes para aplicaciones urbanas en general.

Respecto a la estructura del presente documento, a continuación describimos el área de estudio donde se introduce la selección de los 18 municipios de la Comunidad de Madrid que constituye el Corredor del Henares. La caracterización del área de estudio sirve, además, de justificación para la definición de la estructura básica del modelo que se presenta en el siguiente apartado. El modelo teórico es presentado considerando sus agentes y elementos asociados traducidos en forma de variables de «atracción». En este punto se hace especial hincapié en la cuestión de la disponibilidad de datos teniendo en cuenta las necesidades del modelo, el área de estudio y la escala aplicada. En el apartado IV se presentan los 
componentes particulares que formarían el modelo propuesto, a partir de la especificación de los motivos de su elección y descripción de la relación que tienen con el desarrollo urbano en el área de estudio - lo que vendrá a ser las reglas de comportamiento de los agentes.

\section{III. ÁREA DE ESTUDIO: EL CORREDOR DEL HENARES (COMUNIDAD DE MADRID, ESPAÑA)}

La Comunidad de Madrid (CM), con sus casi $9.000 \mathrm{~km}^{2}$, aglutina una población que se acerca a los 6,5 millones de habitantes, superando los 3 millones el municipio de Madrid, según datos de 2012 (INE, 2014). Debido a su posición estratégica a nivel nacional, concentra funciones importantes y es centro neurálgico de transporte, empleo, administración, entre otros. Estos factores propician una dinámica demográfica activa que, a su vez, refleja cambios territoriales muy significativos.

Su dinámica acelerada de cambios en el uso del suelo se refleja, principalmente, a través de las estadísticas de expansión de suelo urbano y urbanizable, definido por políticas territoriales. No obstante, aunque estas políticas sean un factor decisivo respecto a las dinámicas urbanas, no es el único responsable de la situación de impacto existente. La crisis económica que estalló en 2007 también es un elemento particular que tiene consecuencias territoriales evidentes. En el caso de la CM, el ritmo frenético de la 'industria del ladrillo' se ha minimizado, aunque los impactos generados en el período de expansión inmobiliaria se reflejan en el número espectacular de viviendas vacías y no terminadas. Un ejemplo de ello es el aumento en más de un 200\% del número de viviendas libres iniciadas en la CM entre 1994 y 2004, que pasó de 19.000 a más de 60.000 viviendas, para luego caer a tan sólo 4.600 en los 5 años siguientes (Ministerio de Fomento, 2013).

Por otro lado, este desarrollo urbano acentuado no se dio exactamente en formato tradicional de crecimiento compacto, más bien se distribuyó a lo largo de los ejes de transporte más importantes, principalmente de las carreteras nacionales A-2 y A-4 (que conectan Madrid con Cataluña y con Andalucía, respectivamente). Más recientemente, el eje de la A-6 (que conecta Madrid con Galicia) mostró una evolución urbana destacada de segunda residencia, atraída por la Sierra de Madrid (Hewitt y Escobar Martínez, 2011). Los dos primeros ejes conforman lo que llamamos 'corredores' de expansión urbana - hacia el municipio de Toledo y de Guadalajara (este último, denominado Corredor del Henares).

Debido a las limitaciones de aplicación espacial que todavía tienen los tipos de modelos como el propuesto, se ha seleccionado una parte de la región madrileña como área de estudio, concretamente el Corredor del Henares, que ha experimentado una intensa y compleja dinámica de cambios de usos del suelo en las últimas décadas.

A lo largo de los últimos años, el Corredor del Henares ha sido objeto de estudios realizados por distintos investigadores y organismos públicos. Su delimitación varía significativamente según el organismo que la propone y, principalmente, según el objetivo del estudio. Una revisión de estas distintas delimitaciones muestra un total acuerdo en cuanto a la especificación del eje central, definido por los cuatro municipios principales, que son Alcalá de Henares, Torrejón de Ardoz, San Fernando de Henares y Coslada. Algunas de estas selecciones se basan en análisis puramente temáticos (estadística, movilidad, calidad del aire), mientras otras presentan un perfil más territorial (Gómez Delgado, 1998; López De Lucio, 2003). 
Tras un análisis detallado de las propuestas expuestas, realizamos una nueva selección basándonos en un análisis de dinámica urbana y algunas características de interés como aspectos funcionales, relación con la capital, crecimiento urbano reciente, accesibilidad, etc. La propuesta final de la delimitación del área aglutina 18 municipios (Figura $\mathrm{N}^{\mathrm{o}}$ 1), cuya superficie es de $624 \mathrm{~km}^{2}$, y cuya población asciende a 563.160 habitantes, según los datos del último censo, en 2011 (INE, 2014).

Aunque es sabido que el Corredor del Henares funcionalmente sobrepasa el límite autonómico y presenta una dinámica común y continuada hasta el municipio de Guadalajara (siguiendo el eje de la autopista nacional A-2), ya en otra Comunidad Autónoma (Castilla-La Mancha), nos limitamos a utilizar exclusivamente los municipios de la CM, principalmente debido a la disponibilidad de información necesaria (datos estadísticos y espaciales) para el desarrollo final del modelo, aunque en este trabajo se presente sólo su estructura teórica.

Figura 1

SITUACIÓN Y DELIMITACIÓN DEL CORREDOR DEL HENARES

Y REPRESENTACIÓN DE LOS EJES CENTRAL, NORTE Y SUR
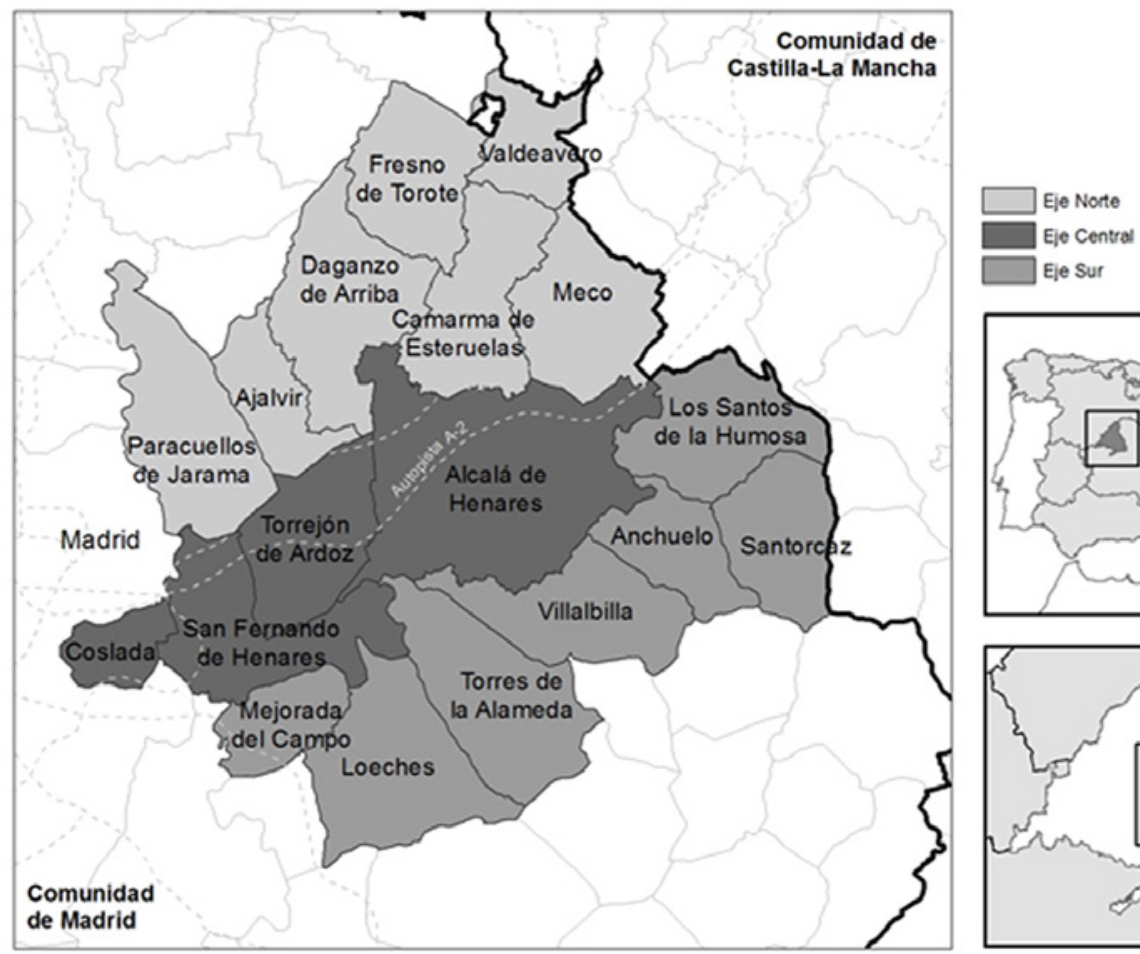

Fuente: elaboración propia.

En general, el desarrollo del Corredor del Henares varía según el período temporal de expansión, y por ello conviene analizarlo por ejes. El eje central siempre ha sido el inductor de básicamente todas las actividades generadas en el área de estudio, y consecuentemente ha 
atraído población y desarrollo urbanístico. En este sentido, dividimos el área de estudio en un eje central que lo corta transversalmente, constituido por los cuatro municipios principales, y dos ejes, norte y sur, que presentan características particulares conforme se presenta más adelante.

El área de estudio experimentó un importante aumento de población en los años de explosión demográfica que siguieron al desarrollismo iniciado en los años 50 del pasado siglo (principalmente en los municipios del eje central, por la oferta de empleo del sector industrial y de servicios). Aunque se observa un incremento constante a partir de este período, destacan dos momentos: entre 1970 y 1981, cuando pasa de 121.790 a 315.663 habitantes, suponiendo un crecimiento del $160 \%$; y luego a partir de 2001, como consecuencia del boom inmobiliario, aumentando un 30\% hasta 2011 (INE, 2011). El primer período de crecimiento se ve frenado por el estallido de la crisis económica y energética, mientras que el segundo lo hace debido a la crisis económica vinculada a la burbuja inmobiliaria.

Así como la estadística de población muestra un crecimiento continuo, también lo muestra la superficie urbanizada. El aumento de esta superficie fue muy intenso entre 1998 y 2006, hecho perfectamente comprobable mediante el análisis de la cartografía de usos del suelo cedida por la Universidad Complutense de Madrid $^{1}$, mostrando un aumento entre 2000 y 2006 de 19,52km² (17,06\%). Aunque la superficie urbana siguió creciendo en el período siguiente, lo hizo en menor proporción - 7,41 km² entre 2006 y 2009 (6,2\%). Esta disminución se debe en gran parte al estallido de la crisis económica y perdura hasta la actualidad.

En cuanto a la distribución de los diferentes usos en el área de estudio, en 2009 (la más actual disponible según la cartografía consultada), dentro de lo que se considera áreas urbanas (ver Cuadro $\mathrm{N}^{\circ} 1$ ), se nota una presencia importante de tejido industrial, así como de áreas residenciales, desarrollo claramente inducido y condicionado por el desarrollo del cercano municipio de Madrid. En cuanto a la morfología de la expansión experimentada, se observa un modelo lineal de desarrollo, confirmando la fuerte influencia de las grandes infraestructuras de comunicación (Serrano Cambronero et al., 2002).

Un análisis por ejes muestra la diferente dinámica de expansión urbana en los períodos que venimos analizando (2000-2006 y 2006-2009). En primer lugar, la expansión del «eje central» antes del año 2000 se encuentra estrechamente ligada a la descongestión del municipio de Madrid. Durante el primer período de análisis (2000-2006) y coincidiendo con el boom inmobiliario, entre los municipios que más crecieron en superficie destacan San Fernando de Henares, que amplió su red de infraestructuras considerablemente; y Alcalá de Henares, con la nueva distribución del área industrial al norte del municipio.

Ya en el «eje norte», es notable en primer lugar el crecimiento de Meco, experimentando casi un $150 \%$ de crecimiento en su superficie urbanizada, seguido de Camarma de Esteruelas y Daganzo de Arriba, también con altos porcentajes (48,6\% y 29,1\%, respectivamente). En el segundo período (2006-2009), ya en fase de descenso de la burbuja inmobiliaria, destaca de nuevo Meco, cuya área urbana sigue ascendiendo (43\%), y Paracuellos de Jarama, que creció casi un $25 \%$.

1 La cartografía de usos del suelo urbano que se utiliza en esta investigación fue cedida por la UCM. Fue creada en el marco de la investigación desarrollada en el Departamento de Geografía e Historia (coordinado por el Prof. Dr. Javier Gutiérrez Puebla) y proporciona información sobre la ocupación del suelo en los años 2000, 2006 y 2009, a escala aproximada 1:10.000. 
Cuadro 1

DISTRIBUCIÓN DE CLASES DEL SUELO EN EL CORREDOR DEL HENARES, 2009

\begin{tabular}{lcc}
\hline \multicolumn{1}{c}{ Clase de usos del suelo } & Superficie $\left(\mathbf{k m}^{\mathbf{2}}\right)$ & Porcentaje respecto al total $\mathbf{( \% )}$ \\
\hline Residencial unifamiliar & 356,8 & 33,48 \\
\hline Residencial multifamiliar & 155,5 & 14,59 \\
\hline Industria & 132,1 & 12,40 \\
\hline Equipamientos & 131,0 & 12,29 \\
\hline Infraestructura & 116,1 & 10,89 \\
\hline Áreas verdes urbanas & 86,4 & 8,11 \\
\hline Aeropuerto & 56,5 & 5,31 \\
\hline Comercio & 19,8 & 1,86 \\
\hline Servicios & 11,6 & 1,08 \\
\hline TOTAL URBANO & $\mathbf{1 . 0 6 5 , 7}$ & $\mathbf{( 1 3 , 2 8 \%}$ del total) \\
\hline TOTAL CORREDOR & $\mathbf{8 . 0 2 5 , 5}$ & \\
\hline
\end{tabular}

Fuente: Cartografía de Usos del Suelo, Dpto. Geografía e Historia - UCM (coord. Javier Gutiérrez Puebla), 2010.

Por último, ya totalmente vinculado a la burbuja inmobiliaria, el «eje sur» experimenta un crecimiento significativo y homogéneo en prácticamente todos los municipios durante el primer período, destacando especialmente Anchuelo (con un incremento del 62,7\%) y Loeches $(54,7 \%)$. De forma general, todos decayeron en el segundo período, registrando apenas crecimiento. La excepción es Villalbilla, que presenta un patrón inverso, con bajo crecimiento en el primer período $(3,4 \%)$ y un $13 \%$ en el segundo.

Basándose en los datos exclusivamente residenciales, se observa que el incremento de $71,8 \mathrm{~km}^{2}$ de áreas residenciales se distribuye espacialmente en las franjas periféricas de los centros urbanos de los municipios, con apenas crecimiento disperso (Figura $\mathrm{N}^{\mathrm{o}} 2$ ).

Por otro lado, el modelo territorial producido por esta ocupación urbana y la distribución poblacional en la CM, están muy relacionados también con el marco legal y las respuestas políticas de ordenación del territorio y urbanismo existentes en España a distintos niveles competenciales. En España, la clasificación del suelo está definida en los Planes Generales de Ordenación Urbana (PGOU), que son competencia única y exclusiva del municipio. Aunque no todos los municipios presenten planes actualizados (según el portal del Sistema de Información Urbana - SIU, en 2012 apenas 8 municipios del área de estudio tenían PGOU, siendo sólo 4 posteriores a 2000), su vigencia en cierto modo estimula el crecimiento en una u otra dirección.

La clasificación del suelo en la Región Urbana de Madrid indica proporciones inquietantes, ya que el porcentaje de suelo urbanizable (12,76\%) equivale a dos tercios del suelo clasificado como urbano consolidado, donde ya viven más de 5,9 millones de habitantes. Ya la misma clasificación para los municipios del Corredor del Henares indica una superficie de $368,48 \mathrm{~km}^{2}$ de área urbanizable delimitada, mientras que $692,56 \mathrm{~km}^{2}$ son áreas urbanas consolidadas. Esto significa que el territorio está legalmente preparado para recibir un $53 \%$ más de la ocupación urbana existente, pudiendo, por lo tanto, duplicar aún su superficie.

Aunque las carencias de los planes regionales (inexistente en la CM) y PGOU en el área de estudio son evidentes, siguen siendo, junto a las leyes de suelo, herramientas fundamenta- 
Figura 2

EXPANSIÓN URBANA EN EL CORREDOR DEL HENARES, 2000-2006-2009
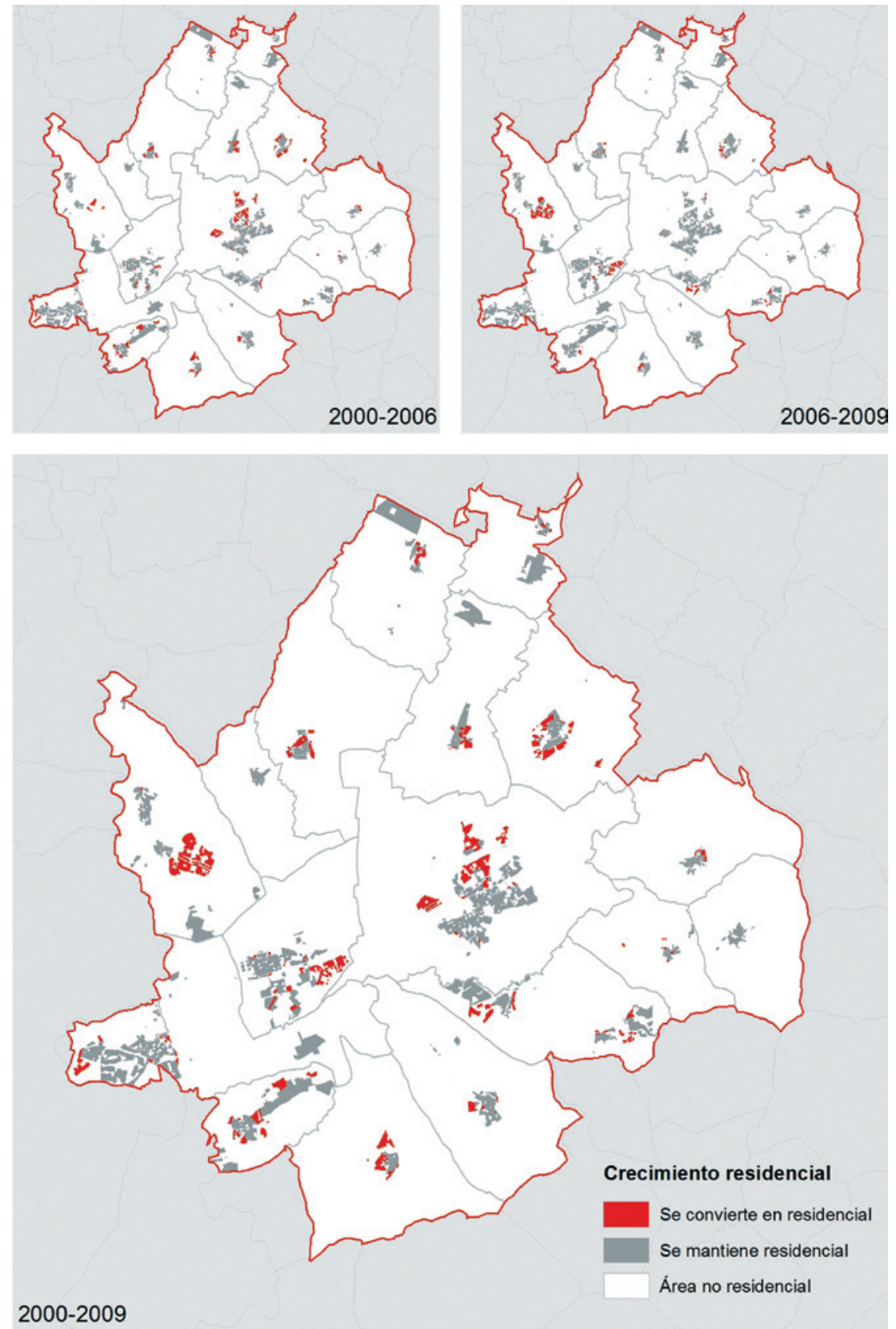

Fuente: Cartografía de Usos del Suelo, Dpto. Geografía e Historia - UCM (coord. Javier Gutiérrez Puebla), 2010. 
les que se encuentran a disposición de la administración y de la comunidad, siendo a su vez elementos esenciales para estudios de crecimiento, tendencias, evolución e impactos de la urbanización sobre el territorio.

Por todo lo expuesto en cuanto al crecimiento demográfico y a las dinámicas de ocupación del suelo (principalmente la expansión de la superficie urbana), así como a la coordinación de este crecimiento con las herramientas ya desfasadas de planificación y ordenación del territorio, y también a los escasos estudios específicos sobre esta región a escala subregional, se considera el Corredor del Henares un área de potencial interés para proceder a realizar un análisis de la expansión urbana con posterior simulación de su crecimiento. De esta forma, se pretende facilitar la comprensión de los elementos espaciales vinculados a su dinámica, así como posibilitar la proyección de las consecuencias de las tomas de decisiones actuales hacia el futuro, acciones altamente valoradas y con gran utilidad en la planificación del territorio.

\section{DISEÑO DE UN MODELO BASADO EN AGENTES PARA LA SIMULACIÓN DEL CRECI- MIENTO URBANO}

El diseño de un MBA debe tener en cuenta la dinámica urbana del área de estudio, que a su vez ayuda a identificar los principales agentes involucrados en el proceso de expansión, así como su comportamiento. Con esto, es posible definir una serie de reglas de decisión que se generarían a partir de información territorial, social y económica.

En este sentido, se resumen a continuación los principales indicadores del proceso de crecimiento urbano experimentado en la Comunidad de Madrid -y consecuentemente también en el Corredor del Henares- en función de una serie de suposiciones y evidencias territoriales.

- La disociación entre crecimiento demográfico y expansión urbana. Aunque en teoría la evolución urbana en un territorio debe estar estrechamente relacionado con el crecimiento demográfico, se observa que, en España en general, y en la Comunidad de Madrid en particular, ambas líneas evolutivas se separaron considerablemente, produciéndose un aumento del suelo urbano no justificado por el aumento de población registrado, sustentado por las elevadas ganancias económicas generadas con el boom inmobiliario a finales de los 90 del pasado siglo y primera mitad de la década de 2000 (OSE - Observatorio de la Sostenibilidad en España, 2010).

- La dinámica acelerada de la promoción inmobiliaria. La promoción de viviendas por parte de pequeños agentes inmobiliarios ha cedido el paso a grandes empresas que necesitan realizar operaciones inmobiliarias importantes para rentabilizar al máximo la inversión de capital (Santos Preciado et al., 2012).

- La influencia del incremento de las infraestructuras de transporte para atender a la población - o para suplir la necesidad generada por las nuevas viviendas en el proceso de crecimiento urbano - es otro de los grandes motivadores del desarrollo urbano. La demanda de transporte público está influenciada por las características de la oferta, sobre las cuales el gestor del transporte puede incidir, y por variables externas (distribuciones de población y empleo, mezcla de usos del suelo, diseño urbano, etc.) (Cardozo et al., 2010). Por otro lado, también es sabido que la mejora y ampliación de 
infraestructuras son determinantes en la expansión de zonas urbanas (Serrano Cambronero, Gago García y Antón Burgos, 2002; López De Lucio, 2003; Plata Rocha, Gómez Delgado y Bosque Sendra, 2010).

- El diseño de nuevos planes generales de ordenación urbana, responsabilidad del sector público municipal y labor de planificadores urbanos, que en teoría trabajan con vistas a un crecimiento controlado y sostenible. Estos planes regulan la estructura y clasificación de los usos del suelo dominantes en la ciudad (residencial, industrial, comercial, etc.), así como los viarios fundamentales y la localización de las principales dotaciones urbanísticas (Santos Preciado et al., 2012).

- Los aspectos legislativos que actúan sobre el uso del suelo son fundamentales para la inducción o retención de la expansión urbana desmedida. La anterior legislación de usos del suelo (Ley 6/1998), de carácter más permisivo, comprometía el territorio protegido, vacío, o con otros tipos de usos no urbanos. La nueva ley del suelo (Ley 8/2007) introduce una serie de modificaciones, entre ellas la caracterización del suelo como recurso a proteger.

- La apertura de medios legales para adquirir préstamos y la facilidad de disponer de créditos financieros, sea por parte de la población o por los promotores inmobiliarios, induce a una fase desarrollista, frenada forzosamente solamente al estallar la crisis financiera.

- El punto anterior se refleja directamente en la dinámica de subida de precios por la compra-venta de inmuebles y alquiler de viviendas, incidiendo en una fuerte especulación inmobiliaria, que a su vez induce a un cambio de perfil de ocupación de alto nivel en regiones específicas, y al mismo tiempo la expulsión a la periferia de la población de rentas más bajas.

- La heterogeneidad en cuanto a la geomorfología en la CM produce intereses adversos en el proceso de expansión urbana. La sierra al noroeste de la comunidad funciona como elemento de atracción y produce una concentración de segunda residencia y urbanizaciones, mientras las áreas más planas del sudeste, aunque tengan características de calidad de suelo menos favorables, inducen la construcción de residencia popular con precios más accesibles, y consecuente ocupación de población de renta más baja.

- La distribución equitativa de equipamientos públicos de educación, salud y ocio es otro elemento que se tiene en cuenta por parte de la población para ubicar su residencia. La proximidad a estos equipamientos puede generar una mayor demanda en determinadas regiones más que en otras.

- La evolución de la red de Espacios Naturales Protegidos (ENP) restringe las áreas de crecimiento urbano, confirmando su rol y sirviendo de protección y conservación de patrimonio natural y paisajístico. Por otro lado, los ENP valorizan (y atraen) áreas urbanas en su vecindad inmediata.

Teniendo en cuenta todo ello, se propone a continuación una estructura conceptual de MBA para simular el crecimiento urbano en el Corredor del Henares, considerando la integración entre la caracterización del fenómeno, el área de estudio, así como los aspectos conceptuales del modelo. Como ya se expuso anteriormente, los MBA se componen bási- 
camente de tres elementos principales: agentes, variables y reglas de comportamiento, definidos en función del área de estudio, del objetivo del modelo y de los datos disponibles. A continuación abordamos cada componente en un apartado independiente, con el objetivo de facilitar la comprensión de lo que son y cómo funcionan, así como para entender mejor cuáles son sus aportaciones al modelo general.

\section{IV.1. Agentes}

Tras una reflexión sobre lo expuesto, y considerando que el primer paso para desarrollar el modelo es definir y concretar qué agentes son aquellos cuya toma de decisiones finalmente podrían producir un cambio real en el territorio en cuanto a la expansión urbana, se presentan los elementos de su estructura funcional. Destacamos que la elección de los agentes considera, entre otras cosas, (1) el histórico cultural del área de estudio, que en este caso indica la fuerte relación con Madrid, (2) el fenómeno estudiado de expansión urbana acelerada, (3) la escala que toma como referencia el ámbito subregional, así como, y principalmente, (4) el enfoque que se quiere dar a la modelación, que vienen a ser simulaciones que sirvan de apoyo a la planificación urbana, y como se ha presentado en la introducción de este documento, como una herramienta exploratoria para simular diferentes propuestas de futuro.

$\mathrm{Al}$ elegir una escala subregional para aplicar el modelo, el abanico de opciones de tipos de agentes se restringe considerablemente. Por ejemplo, los agentes no serán configurados como individuos independientes, sino como una agregación de individuos con características similares.

Integrando las suposiciones expuestas definimos finalmente los tres agentes: (1) la población, (2) los promotores inmobiliarios y (3) los planificadores urbanos. En esta propuesta entendemos que los tres grupos estimulan paralelamente este fenómeno de acuerdo a una serie de elementos que inducen o limitan la ocupación urbana sobre el territorio (Cantergiani, 2011b).

La influencia de estos tres agentes sobre el desarrollo urbano se basa en relaciones de oferta y demanda de áreas urbanizables, edificación residencial y cercanía o ampliación de otros elementos de interés como infraestructuras, equipamientos urbanos o áreas verdes. Sus acciones se configuran en un ciclo que, aunque no genera elementos de competición directa, se retroalimentan según cómo reaccione uno u otro agente o grupo de agentes. Esto es, los planificadores asignan nuevo suelo urbanizable (teniendo en cuenta aquél territorio que todavía no está urbanizado); los promotores urbanos buscan, dentro de las áreas clasificadas como urbanizables, áreas de interés para nueva construcción de viviendas (según los intereses del mercado); por último la población elige su lugar de residencia (considerando la oferta de viviendas nuevas y vacías).

En este sentido, la población actúa como el agente motor que decide cambiar de residencia y transformar el territorio, generando la demanda de nuevas viviendas y consecuente expansión urbana. El agente población será distribuido en tres grupos que difieren considerablemente en cuanto a las decisiones que subyacen la elección del nuevo lugar de residencia (en función de sus intereses y necesidades). En la literatura de modelación urbana podemos encontrar diversos ejemplos de agrupaciones de agentes, según la edad (Fokkema y Vanwissen, 1997) o ciclos de vida (soltero, casado sin hijos, casado con hijos, jubilado, etc.) (Die- 
leman, 2001; Fontaine y Rounsevell, 2009; Jordan, Birkin y Evans, 2012). En nuestro caso, y por las características del modelo y los agentes implicados, se ha decidido establecer una diferenciación en función del poder adquisitivo familiar (nivel de renta alta, media o baja), cuya agrupación también comparten otros autores (Barros, 2004; Aguilera y Ugalde, 2007).

Por otro lado, los promotores inmobiliarios son aquellos actores que promueven la construcción de nuevas viviendas. Los promotores inmobiliarios podrían actuar según la situación económica de la empresa, estatus o experiencia de mercado, entre otros. Sin embargo, en el modelo asumimos que todos los promotores inmobiliarios presentarán idéntico comportamiento, y por ello se representarán a través de un único tipo.

Por último, el tercer agente del modelo son los planificadores urbanos (PU), con un importante rol en la elaboración de Planes Urbanísticos y de Ordenación del Territorio. Para los planificadores urbanos sería posible diferenciar su comportamiento según la orientación política o directrices urbanísticas, por ejemplo, sobre todo considerando el nivel municipal, pudiéndose presentar en cada municipio un comportamiento distinto. No obstante, al igual que para los promotores inmobiliarios, asumiremos que los planificadores urbanos presentan un único comportamiento.

\section{IV.2 Variables}

Además de especificar cuáles son los agentes que interactúan entre ellos y con el medio, hace falta definir claramente una serie de reglas que guíen el comportamiento de cada agente y agrupaciones. Estas reglas deben representar el proceso de toma de decisiones de cada tipo de agente según las suposiciones consideradas. Para ello, es fundamental tener en cuenta los principales intereses espaciales de cada uno y los factores que les motivan a actuar de una forma u otra.

A continuación, se presenta un listado de lo que consideramos serían los principales elementos de atracción (espaciales y no espaciales) asignados a cada uno de los tres agentes planteados, teniendo en cuenta el área de estudio (Cuadro $\mathrm{N}^{\circ} 2$ ). Para la elaboración de este listado, que siempre resultará incompleto, se ha tomado como referencia la revisión de varios trabajos en los que se definen de alguna forma variables de atracción para el crecimiento urbano (Plata Rocha et al., 2009), así como el conocimiento del área de estudio por parte de los expertos involucrados en esta investigación (Proyecto SIMURBAN²).

Aunque el objetivo del trabajo es presentar tan sólo la estructura teórica del modelo, creemos conveniente hacer alguna alusión a la dificultad que puede suponer (y que está suponiendo) el desarrollo práctico del modelo. La traducción del modelo teórico al modelo práctico en la etapa de implementación sugiere una serie de reconsideraciones y acotaciones en cuanto a la definición de los elementos de atracción de cada tipo. Por supuesto que la selección de estos elementos seguirán los intereses particulares de los agentes en cada situación, conforme no sólo a sus preferencias, sino también a la disponibilidad de la información necesaria que, en nuestro caso, es limitada debido a la escala propuesta para el desarrollo del modelo y al área de estudio elegida.

2 Proyecto SIMURBAN: Análisis y simulación prospectiva mediante Tecnologías de la Información Geográfica (TIG) del crecimiento urbano actual. Evaluación de su sostenibilidad. http://www.geogra.uah.es/simurban1/ (acceso en Julio/2014) 
Cuadro 2

AGENTES Y ELEMENTOS DE ATRACCIÓN ESPACIAL

\begin{tabular}{|c|c|}
\hline Agente & Elementos de atracción \\
\hline Población & $\begin{array}{l}\text { - Existencia de viviendas libres y/o nuevas } \\
\text { o infraestructura viaria } \\
\text { o accesibilidad al transporte público } \\
\text { o centros comerciales } \\
\text { o áreas urbanas consolidadas } \\
\text { o equipamientos públicos de salud y educación } \\
\text { - Precio de la vivienda accesible de acuerdo a la renta de cada grupo } \\
\text { - Proximidad a grupos sociales semejantes } \\
\text { - Calidad de vida - cercanía a parques urbanos y espacios de ocio }\end{array}$ \\
\hline $\begin{array}{l}\text { Promotores } \\
\text { inmobiliarios }\end{array}$ & $\begin{array}{l}\text { Disponibilidad de terrenos para urbanizar, obedeciendo a: } \\
\text { o clasificación de suelo apropiada (según legislación vigente) } \\
\text { o geotecnia adecuada para la construcción } \\
\text { - Configuración territorial atractiva para la población } \\
\text { o accesibilidad y movilidad (infraestructuras y transporte público) } \\
\text { o densidad de edificación } \\
\text { - Valoración del suelo accesible de acuerdo a los medios económicos } \\
\text { - Cercanía a áreas urbanas consolidadas }\end{array}$ \\
\hline $\begin{array}{l}\text { Planificadores } \\
\text { urbanos }\end{array}$ & $\begin{array}{l}\text { - Disponibilidad de terrenos no productivos } \\
\text { - Cercanía a: } \\
\text { o áreas urbanas consolidadas } \\
\text { o infraestructura viaria } \\
\text { o equipamientos públicos de salud y educación } \\
\text { - Disponibilidad de áreas sin protección legal (ENP, redes hidrográficas) } \\
\text { - Lejanía a equipamientos molestos y peligrosos (no deseables) }\end{array}$ \\
\hline
\end{tabular}

Fuente: elaboración propia.

Para que se puedan realizar los análisis propuestos, y basándonos en las variables expuestas, es necesario un trabajo exhaustivo de compilación de esta información. Hemos de destacar la importancia de los datos espaciales, dado que el modelo es dinámico y se basa en la distribución de sus elementos y características en forma de celdas que interactúan entre ellas y generan una nueva respuesta espacial. Los Sistemas de Información Geográfica (SIG) son herramientas fundamentales para elaborar una base de datos de referencia para el modelo, que será consultada internamente por los agentes.

En nuestro caso concreto, de modo general, se puede afirmar que estas bases de datos de referencia para la Comunidad de Madrid son relativamente amplias, y están, en su mayoría, disponibles a través de organismos oficiales estatales o autonómicos ${ }^{3}$. En este sentido, hemos

3 Instituto Geográfico Nacional (IGN), Centro Nacional de Información Geográfica (CNIG), Ministerio de Agricultura, Alimentación y Medio Ambiente (MAGRAMA), Dirección General de Catastro, Instituto de Estadística de la Comunidad de Madrid 
de destacar el esfuerzo que la administración pública ha realizado en los últimos años para facilitar el acceso a una importante cantidad de información espacial y estadística.

Sin embargo, es evidente que no todos los datos disponibles se adaptan perfectamente al modelo diseñado, por lo que es necesario un análisis escrupuloso de los datos existentes en función del modelo propuesto y de los resultados esperados. También hemos de matizar que desafortunadamente existe una amplia cantidad de información oficial que no se encuentra todavía disponible para uso externo a los organismos productores, y cuya incorporación al modelo hubiera sido fundamental. Es el caso de la información económica sobre renta, precios de vivienda y valor del suelo, necesarios para estudiar y simular adecuadamente el comportamiento de la población o de los promotores inmobiliarios, por ejemplo.

Con el objetivo de integrar tantos datos espaciales en un MBA, con información a escala subregional, se hizo necesaria una serie de ajustes, estimaciones, adaptaciones y suposiciones sobre los pocos datos disponibles, principalmente en lo que concierne a la integración de datos socioeconómicos. Además de estos, se incorporaron otros más básicos, como es el caso del número de viviendas por sección censal, que aunque pareciera una tarea sencilla, también supuso un esfuerzo importante de geoprocesamiento.

\section{IV.3. Reglas de comportamiento}

Tras definir los agentes, identificar su estado de insatisfacción, necesidad o interés (que como consecuencia induce el cambio a simular), reconocer la disponibilidad interna y externa para la materialización de este posible cambio, e identificar los elementos de atracción espacial y datos disponibles, faltaría definir las reglas de comportamiento para concluir el diseño del modelo teórico-conceptual. Todos estos elementos se complementan y actúan de forma integrada, a su vez reflejando el comportamiento de cada agente y del sistema, lo que produce finalmente los resultados de la simulación.

La traducción del modelo teórico presentado en los apartados anteriores al modelo conceptual, ya teniendo en cuenta su aplicación para el Corredor del Henares y considerando los datos disponibles, exige naturalmente una serie de pequeñas adaptaciones. De esta forma, se presenta a continuación y para cada agente la elección final de los elementos para la toma de decisiones y de los componentes de atracción que se utilizarán en la implementación del modelo. Cabe destacar que la estructura de acción es la misma para todos (Figura $\mathrm{N}^{\mathrm{o}} 3$ ), es decir, partiendo de una situación inicial con unas determinadas restricciones (A), que a través de la interacción con el medio y de los intereses propios (B), alcanza un estado final donde se refleja el cambio producido. Por la dinámica intrínseca de los MBA, el estado final vuelve a actuar como estado inicial en la etapa subsecuente.

En el caso del proceso de los agentes planificadores urbanos, que optan por asignar (o no) nuevas áreas urbanizables, el agente parte de una clasificación inicial de usos del suelo (urbano, urbanizable, otros). Como restricción (A), considera todo aquello que no se podrá asignar como nuevo suelo urbanizable (por ejemplo, una determinada franja a lo largo de los ríos, Espacios Naturales Protegidos, las infraestructuras de transporte, zonas con pendientes superiores a $10 \%$ y áreas ya urbanas o urbanizables). Los criterios de interés (B) para estos agentes se materializan en los siguientes factores espaciales: cercanía a carreteras y equipamientos públicos, distancia a instalaciones no deseables y/o peligrosas (vertederos, gasoduc- 
Figura 3

ESQUEMA DEL PROCESO DE DECISIÓN DE LOS AGENTES

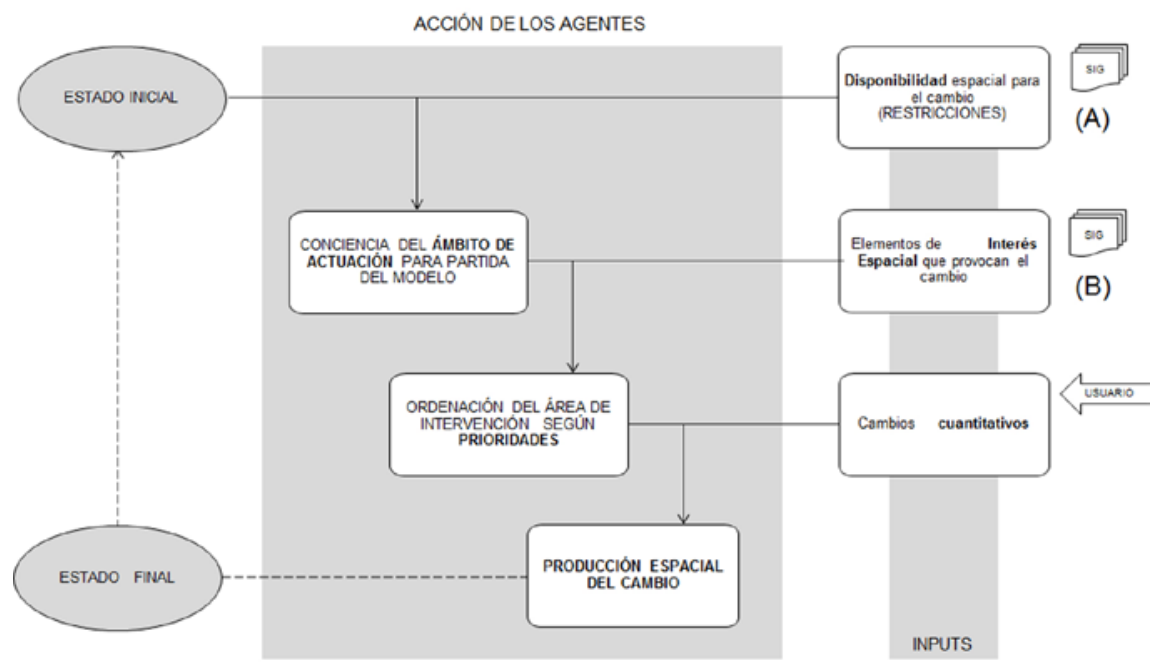

Fuente: elaboración propia.

tos, cárceles, plantas de tratamiento de residuos, etc.), productividad del terreno (buscan no ocupar terrenos con alta productividad agraria), existencia de áreas urbanas consolidadas (con el objetivo de optimizar la utilización de recursos públicos relacionados con servicios a la población de seguridad, transporte, equipamientos urbanos), que según la dinámica histórica del Corredor del Henares pueden representar el comportamiento general de los planificadores urbanos (Figura $\mathrm{N}^{\mathrm{0}} 4$ ).

Para el segundo agente, los promotores inmobiliarios, el esquema es similar. El objetivo en este caso es la construcción de nuevas residencias en áreas asignadas como urbanizables, lo que ya indica la restricción espacial (A). En este caso, la construcción puede enfocarse a diferentes niveles de construcción, de alto, medio o bajo estándar, según la situación del mercado en el momento de la decisión. Esto introduce un factor de aleatoriedad al modelo, ya que el orden del tipo de construcción prioritario puede variar. Como factores espaciales de atracción (B), se consideran: cercanía a carreteras, equipamientos de transporte público y áreas urbanas consolidadas, la densidad de edificación, y principalmente el valor del suelo (Figura $\mathrm{N}^{\circ}$ 5).

Por último, el comportamiento de la población difiere según el grupo social al que pertenezca, definido por rangos medios de renta, lo que añade una cierta complejidad a estos agentes. Se parte de la oferta de viviendas vacías (libres o nuevas) y del nivel de renta de su potencial morador (A). Debido a esta agrupación de los agentes, no sólo no coinciden en la preferencia en cuanto a algunos factores espaciales (la cercanía al transporte público será importante para la población de renta baja, pero no lo será, por ejemplo, para los grupos de renta alta), sino que también pueden tener reacciones distintas en función de la misma variable (B). Es el caso del precio de la vivienda, que presenta restricciones fuertes para algunos grupos de población y débiles para otros. Los demás factores considerados por la población 
Figura 4

MODELO CONCEPTUAL DE DECISIÓN DEL AGENTE PLANIFICADOR URBANO

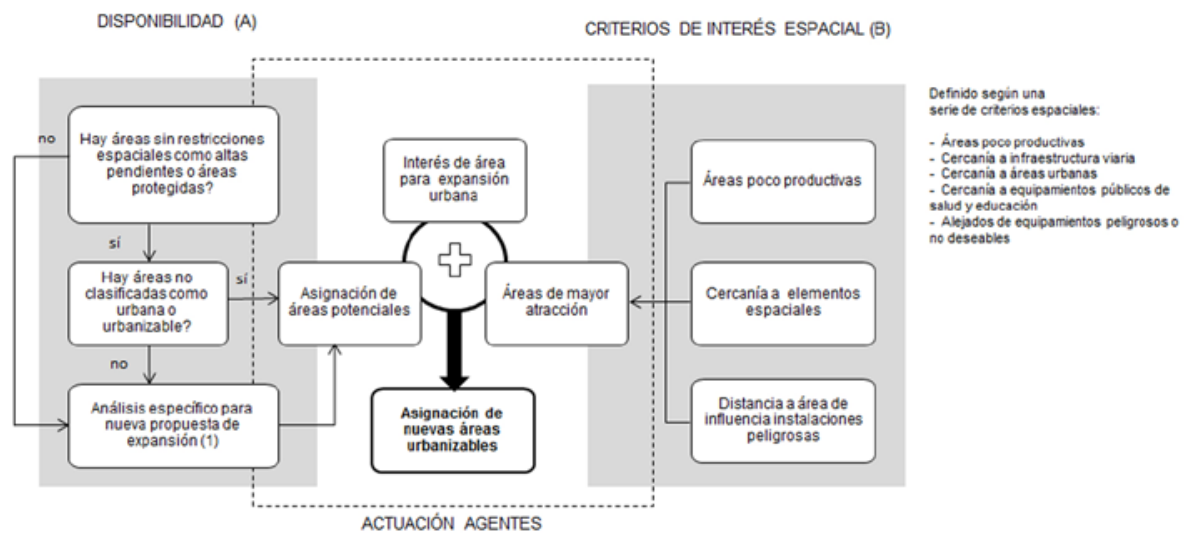

(1) Nuevas propuestas de amplación de áreas urbanizables en regiones no inicialmente planteadas (ej. creadón de urbanizaciones aislados, ocupación de áreas con determinada restriccion)

Fuente: elaboración propia.

Figura 5

MODELO CONCEPTUAL DE DECISIÓN DEL AGENTE PROMOTOR INMOBILIARIO

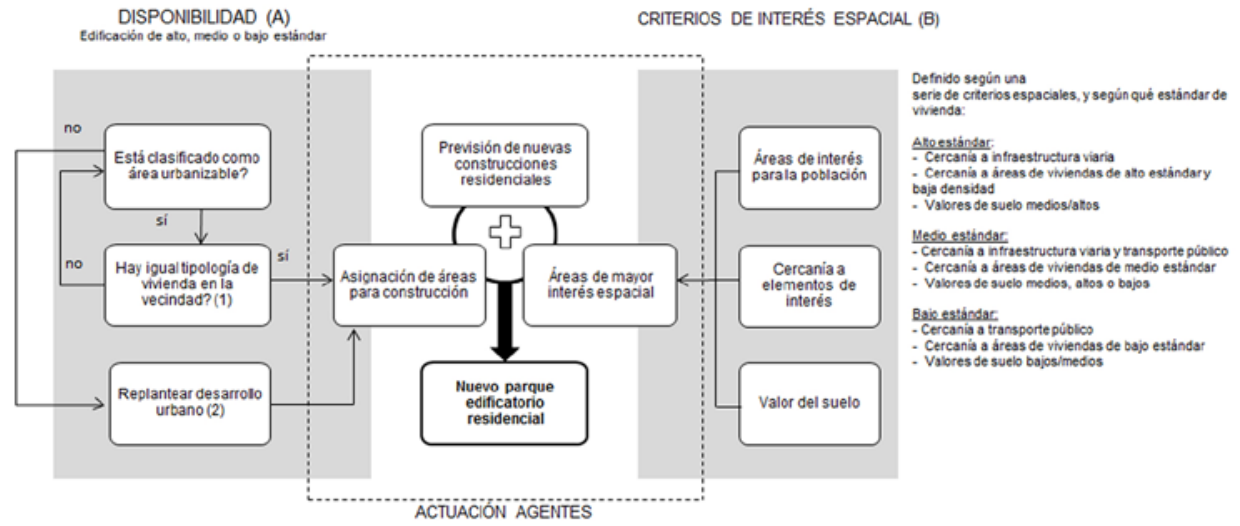

(1) Excepto para construcción de viviendas de alto estándar, cuando hay posibilidad de generar urbancaciones aistadas

(2) Por ejemplo, desarrollo interiox

Fuente: elaboración propia.

son: cercanía a carreteras/transporte público/centros comerciales/equipamientos de salud y educación, el nivel de renta de la vecindad y el precio medio de la vivienda, siempre teniendo en cuenta el nivel económico de la población (Figura $\mathrm{N}^{\mathrm{0}} 6$ ).

La implementación del modelo, en un primer momento, cuenta con una cantidad reducida de datos, pues sólo así es posible comprender cómo evoluciona el modelo en cada etapa 
y conocer en profundidad cómo funciona la interacción de los agentes y las variables que pueden incidir en su comportamiento. Además, la menor cantidad de elementos en el modelo facilita la detección de posibles inconsistencias técnicas de programación en su ejecución, en una primera etapa de desarrollo del mismo.

En cualquier caso, aunque la cantidad de datos sea reducida, el desarrollo del MBA integrado resultará complejo, considerando que integra las reacciones de los tres agentes cuyos submodelos se desarrollan de forma separada en una primera etapa. El modelo integrado contará con la incorporación de acciones y reacciones de un agente como información de partida o restricción para otro agente (Figura $\mathrm{N}^{\mathrm{o}} 7$ ).

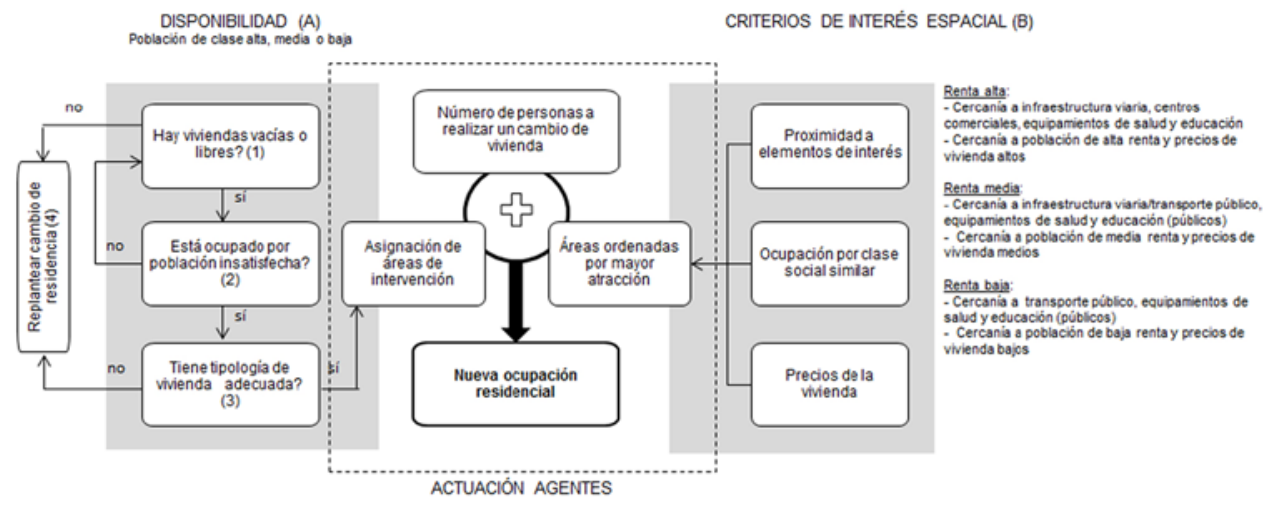

(1) Viviendas libres son aquellas cuya ocupación no llega a la capacidad máxima, o sea, todavia con espacio disponible para ocupación (2) La población que desea cambiar de residencia (insatistecha). y con renta inferior a de la que buscavivienda

(3) La tipología adecuada es aquella de igual nivel o con un nivel de diferencia (para pobladón de renta alta, viviendas de alto o medio estándar, para podlación de renta media, cualquier tpologia de vinenda, parapoblacion de renta caja, nsiendas de mecio (paj estándar)

(4) Replantear la necesidad de cambiarse de residencia o plantear ocuparviviendas con otra tipologia

Fuente: elaboración propia.

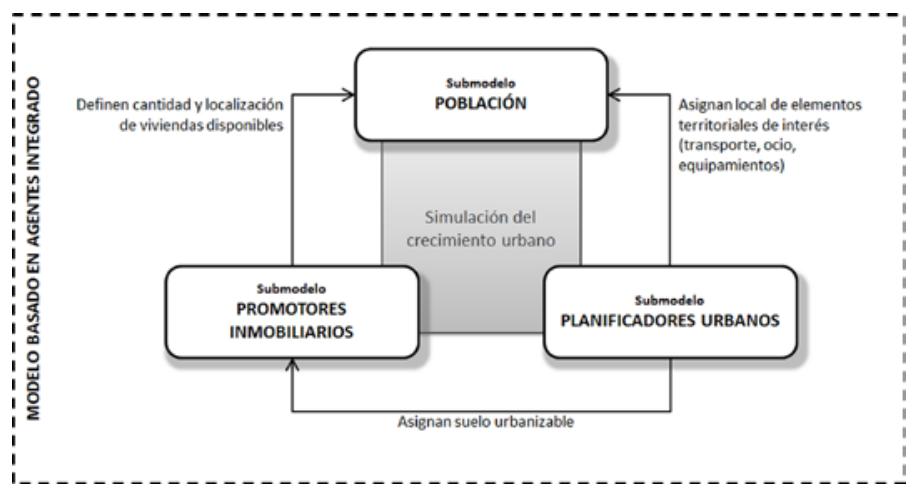

Fuente: elaboración propia. 
En resumen, este modelo integrado consta de los tres sub-modelos expuestos ejecutándose de forma casi simultánea, en el que el producto de la acción de los planificadores urbanos -las áreas urbanizables- alimenta o limita la actuación de los promotores inmobiliarios, que a su vez construyen nuevas residencias que se ofertarán a la población. En un movimiento dinámico y continuo, el modelo simula paso a paso el crecimiento urbano del Corredor del Henares, cumpliendo el objetivo inicial propuesto en la construcción del MBA.

\section{REFLEXIÓN FINAL Y CONCLUSIONES}

Partimos de la idea de que los MBA podrían ser empleados para simular el crecimiento urbano a una escala subregional. La elaboración del mismo, debe pasar inexcusablemente por el desarrollo de un cuidadoso diseño. La compleción de una serie de etapas conceptuales previas al desarrollo del modelo es positiva y necesaria en el sentido de que permite situarlo dentro de un contexto de simulación, además de organizar y ordenar las decisiones en cuanto a su estructura. Aunque resulte muy descriptivo en algunas fases, sirve de base de referencia y es fundamental para la futura implementación del MBA y su programación.

Los agentes elegidos (población, promotores inmobiliarios y planificadores urbanos), pensamos que reflejan adecuadamente el conjunto de actores implicados en el proceso de localización del crecimiento urbano en un territorio como el Corredor del Henares. Las variables de atracción seleccionadas se basan principalmente en vínculos territoriales que pueden indicar mejor calidad de vida u optimización de recursos (tiempo o dinero), tal como cercanía a infraestructuras viarias, transporte público o equipamientos urbanos. Sin embargo, en un MBA que destaca por la posibilidad de reproducir comportamientos, hay otros elementos importantes a considerar, como los socioeconómicos (relación de vecindad y renta, precios de vivienda y valor del suelo), principalmente cuando se trata de aplicaciones urbanas. La consideración de estos elementos es un diferencial del presente modelo.

Asimismo, la definición inicial de los componentes del MBA (agentes, variables de atracción y reglas de comportamiento) a la escala subregional presentada es novedosa, ya que los MBA suelen aplicarse a una escala más local cuando se trata de un enfoque urbano. Aunque este modelo podría aplicarse en otras áreas urbanas en España, y quizás fuera de ella, aquí la elección de cada componente se define en función del desarrollo acelerado que se observa concretamente en este área de estudio en las últimas décadas, pero debería ser cuidadosamente revisado en cada nuevo objetivo, escala o contexto geográfico, político, social y cultural en los que pretenda aplicar.

En el caso del trabajo presentado, destacamos que los 18 municipios seleccionados para constituir el área de estudio presentan un eje central muy dinámico urbanísticamente, que debido a las elevadas tasas de crecimiento urbano en la última década, se transforma en un área de especial interés para la simulación, incrementado por la ausencia de una planificación a nivel regional. Hemos de tener en cuenta que se trata de un territorio que ha registrado una tendencia de crecimiento continuo (aunque minimizado al estallar la crisis económica) y todavía existen abundantes terrenos disponibles para la urbanización en gran parte de los municipios del área de estudio, por lo que resulta todavía de mayor interés la posibilidad de contar con herramientas de simulación para apoyar las posibles iniciativas en este sentido, y así realizar una correcta planificación de este espacio. 
No obstante, la falta de alguna información espacial y alfanumérica de entrada al nivel de detalle necesario, y la imposibilidad de disponer de datos públicos no compartidos con la comunidad científica (y menos con el público general), puede dificultar la elaboración del «mejor» modelo. Como hemos mencionado, en el caso del Corredor del Henares toda la información socioeconómica ha tenido que ser sometida a una serie de procesamientos espaciales que, aunque permitan la ejecución del modelo con datos de menor precisión, producen ciertas generalizaciones que pueden introducir incertidumbre. En este sentido, hemos de mencionar el papel relevante que tienen los SIG en esta tarea, facilitando la gestión y tratamiento de la información. No obstante, la conexión con las plataformas utilizadas para el desarrollo de estos modelos todavía se encuentra sin resolver de manera óptima.

En cualquier caso, independientemente de los problemas que se puedan encontrar al proponer el diseño de un MBA a escala subregional (con apenas referencias científicas en la actualidad), el diseño que se presenta permite introducir los puntos más importantes y fundamentales de la estructura de un MBA, así como detectar dificultades y plantear posibles soluciones a esas deficiencias. Además de ser un aporte teórico, y quizás una especie de guía de cómo elaborar un MBA desde cero, es también una semilla para una aplicación práctica que ya se encuentra en desarrollo, y que en breve se difundirá en el medio científico (productos de los submodelos independientes, así como del modelo integrado).

En suma, el presente documento puede ser útil como fuente de referencia e información para aplicaciones similares en otras áreas de estudio, siempre teniendo en cuenta las discrepancias en cuando al objetivo, singularidades del área de estudio, escala y datos disponibles. Ante todas las consideraciones expuestas a lo largo de este documento, creemos que los MBA pueden ser interesantes para la simulación del crecimiento urbano, ya que la información mínima necesaria puede ser compilada e integrada en el modelo, y utilizada en la definición de las reglas de comportamiento de los agentes, llegando más allá de los cambios territoriales exclusivamente de vecindad de los AC, que aunque presentan indudables potencialidades, están limitados por la falta de movilidad y dinamismo que sí aportan los agentes al sistema. Aun así, sabemos que todavía hay un largo camino por recorrer para demostrar la eficacia de estos modelos en este ámbito.

\section{AGRADECIMIENTOS}

La presente investigación se desarrolla en el marco del proyecto «Instrumentos de Geosimulación y planificación ambiental en la ordenación territorial de ámbitos metropolitanos. Aplicación a escalas intermedias (SIMURBAN2)», financiado por el Ministerio de Economía y Competitividad del Gobierno de España (Ref.: CSO2012-38158-C02-01).

\section{BIBLIOGRAFÍA}

AGUILERA, A. y UGALDE, E. (2007): «A spatially extended model for residential segregation». Discrete Dynamics in Nature and Society. Disponible en http://arxiv.org/pdf/ nlin/0607026.pdf (acceso en Julio 2014). 
AGUILERA BENAVENTE, F., GÓMEZ DELGADO, M. y CANTERGIANI, C.C. (2010): «Instrumentos de simulación prospectiva del crecimiento urbano». Ciudad y Territorio, $\mathrm{n}^{\circ} 165-166,481-496$.

AGUILERA BENAVENTE, F.,VALENZUELA MONTES, L.M. y BOSQUE SENDRA, J. (2010): «Simulación de escenarios futuros en la aglomeración urbana de Granada a través de modelos basados en Autómatas Celulares». Boletín de la AGE, n 54, 271-300.

BARREDO CANO, J.I. y GÓMEZ DELGADO, M. (2008): «Towards a set of IPCC SRES urban land-use scenarios: modelling urban land-use in the Madrid region». En M. PAEGELOW y M.T. CAMACHO OLMEDO (eds.): Modelling Environmental Dynamics: Advances in Geomatic Solutions (págs. 365-385), Springer.

BARROS, J. (2004): Urban Growth in Latin American Cities. Exploring urban dynamics through agent-based simulation. Tesis doctoral inédita, Bartlett School of Graduate Studies, Bartlett School of Architecture and Planning, University College London, University of London, London.

BATTY, M. (2005): Cities and Complexity: understanding cities with cellular automata, agent-based models, and fractals, Massachusetts Institute of Technology.

BENENSON, I.,OMER, I. y HATNA, E. (2002): «Entity-based modeling of urban residential dynamics: the case of Yaffo, Tel Aviv». Environment and Planning B, $\mathrm{n}^{\circ}$ 29(4), 491-512.

BENENSON, I. y TORRENS, P.M. (2004): Geosimulation: Automata-based modeling or urban phenomena, John Wiley \& Sons, L.

CANTERGIANI, C.C. (2011a): «Modelos basados en agentes aplicados a estudios urbanos: una aproximación teórica». Serie Geográfica, n 17, 29-43.

CANTERGIANI, C.C. (2011b): «Identificación de componentes espaciales en un Modelo Basado en Agentes». En N.N. PINTO et al. (Eds.): 7th Virtual Cities and Territories (págs. 65-69). Lisbon.

CARDOZO, O.D., GUTIÉRREZ PUEBLA, J. y GARCÍA PALOMARES, J.C. (2010): «Influencia de la morfología urbana en la demanda de transporte público: análisis mediante SIG y modelos de regresión múltiple». Geofocus, n 10, 82-102.

CARSJENS, G.J. (2009): Supporting Strategic Spatial Planning. Planning Support Systems for the spatial planning of metropolitan landscapes. Tesis doctoral inédita, Universidad de Wageningen, Holanda.

CASTLE, C.J.E. (2007): «Guidelines to Assess Pedestrian Evacuation Software Applications». UCL Working Papers Series, 115.

CASTLE, C.J.E. y CROOKS, A.T. (2006): «Principles and Concepts of Agent-Based Modelling for Developing Geospatial Simulations». UCL Working Papers Series, 110. Disponible.

CROOKS, A.T. (2006): «Exploring Cities Using Agent-Based Models and GIS». UCL Working Papers Series, 109.

DEARDEN, J. y WILSON, A. (2012): «The Relationship of Dynamic Entropy Maximising and Agent-Based Approaches in Urban Modelling». En A. HEPPENSTALL et al. (eds.): Agent-Based Models of Geographical Systems (págs. 705-720), Springer.

DIELEMAN, F. (2001): «Modelling residential mobility: A review of recent trends in research». Journal of Housing and the Built Environment, $\mathrm{n}^{\circ}$ 16(3), 249-265. 
ENCINAS, M.A., HERNÁNDEZ-JIMÉNEZ, V., HERNÁNDEZ, C., MARTÍNEZ, L. y WINDER, N. (2006): A Conceptual Model of Land Use Change in the Madrid Autonomous Region. Informe No. TiGRESS Final Repor / FPV EC.

EPSTEIN, J.M. y AXTELL, R. (1996): Growing Artificial Societies: Social Science from the Bottom Up. Washington D.C., MIT Press.

ETTEMA, D. (2011): «A multi-agent model of urban processes: Modelling relocation processes and price setting in housing markets». Computers, Environment and Urban Systems, $\mathrm{n}^{\mathrm{o}} 35(1), 1-11$.

FEITOSA, F.F., LE, Q.B. y VLEK, P.L.G. (2011): «Multi-agent simulator for urban segregation (MASUS): A tool to explore alternatives for promoting inclusive cities». Computers, Environment and Urban Systems, $\mathrm{n}^{\mathrm{o}} 35(2), 104-115$.

FILATOVA, T., PARKER, D.C. y VAN DER VEEN, A. (2009): «Agent-Based Urban Land Markets: Agent's princing behavior, land prices and urban land use change». Journal of Artificial Societies and Social Simulation, 12 (1)(3).

FOKKEMA, T. y VANWISSEN, L. (1997): «Moving plans of the elderly: a test of the stressthreshold model». Environment and Planning A, n 29(2), 249-268.

FONTAINE, C.M. y ROUNSEVELL, M.D.A. (2009): «An agent-based approach to model futures residential pressure on a regional landscape». Landscape Ecology, n 24(9), 12371254.

GALLARDO, M. y MARTÍNEZ-VEGA, J. (2012): «Cambios de usos del suelo en la Comunidad de Madrid: Analizando el pasado y simulando el futuro». En XV Congreso Nacional de Tecnologías de la Información Geográfica. Madrid, AGE-CSIC.

GILBERT, N. y TROITZSCH, K.G. (2005): Simulation for the Social Scientist (second ed.). Glasgow, Open University Press.

GÓMEZ DELGADO, M. (1998): Análisis geográfico de la producción y gestión de residuos tóxicos y peligrosos en el Corredor Industrial del Henares. Cálculo de rutas óptimas para su transporte mediante un SIG. Tesis doctoral inédita, Universidad de Alcalá de Henares, Alcalá de Henares.

GÓMEZ DELGADO, M. y BARREDO CANO, J.I. (2005): Sistemas de Información Geográfica y Evaluación Multicriterio. Paracuellos de Jarama, RA-MA.

GRIMM, V., BERGER, U.,BASTIANSEN, F., ELIASSEN, S., GINOT, V., GISKE, J.,GOSS-CUSTARD, J., GRAND, T., HEINZ, S.K., HUSE, G., HUTH, A., JEPSEN, J.U., JORGENSEN, C., MOOIJ, W.M. y MULLER (2006): «A standard protocol for describing individual-based and agent-based models». Ecology Modelling, n 198, 115-126.

HANSEN, H.S. (2010): «Modelling the futures coastal zone urban development as implied by the IPCC SRES and assessing the impact from sea level rise». Landscape and Urban Planning, $\mathrm{n}^{\circ}$ 98, 141-149.

HEPPENSTALL, A.,CROOKS, A.T.,SEE, L.M. y BATTY, M. (2012): Agent-Based Models of Geographical Systems. Dordrecht, Springer.

HEWITT, R. y ESCOBAR MARTÍNEZ, F. (2011): «The territorial dynamics of fast-growing regions: Unsustainable land use change and future policy challenges in Madrid, Spain». Applied Geography, n 31, 650-667.

INE Instituto Nacional de Estadística (2014), Datos de padrón y censo disponibles en http:// www.ine.es (acceso en Julio 2014). 
JIANG, B. (2000): «Agent-based approach to modeling urban and environmental systems within GIS». En 9th International Symposium on Spatial Data Handling.

JORDAN, R., BIRKIN, M. y EVANS, A. (2012): «Agent-Based Modeling of Residential Mobility, Housing Choice and Regeneration». En A. HEPPENSTALL et al. (eds.): Agent-Based Models of Geographical Systems (págs. 511-524), Springer.

LÓPEZ DE LUCIO, R. (2003): «Transformaciones territoriales recientes en la región urbana de Madrid». Urban, $\mathrm{n}^{\circ}$ 8, 124-161.

LÓPEZ, E.,BOCCO, G., MENDOZA, M. y DUHAU, E. (2001): «Predicting land-cover and land-use change in the urban fringe. A Case in Morelia City, Mexico». Landscape and Urban Planning, $\mathrm{n}^{\circ}$ 55, 271-285.

MINISTERIO DE FOMENTO (2013). Información estadística sobre viviendas libres. Disponible en http://www.fomento.gob.es/BE2/?nivel=2\&orden=32000000 (acceso en Julio 2014).

NORTE PINTO, N.E.,DOURADO, J. y NATÁLIO, A. (2012, November 8-10 2012): «Cellular Automata Modeling for Urban and Spatial Systems». En The International Symposium on Cellular Automata Modeling for Urban and Spatial Systems - CAMUSS. Oporto.

OSE - OBSERVATORIO DE LA SOSTENIBILIDAD EN ESPAÑA (2010): Informe Sostenibilidad en España 2010. Informe. Madrid, Observatorio de la Sostenibilidad en España - OSE.

PAEGELOW, M. y CAMACHO OLMEDO, M.T. (2008): Modelling environmental dynamics. Berlin, Springer-Verlag.

PARKER, D.C., BERGER, T. y MANSON, S.M. (2001): «Agent-Based Models of LandUse and Land-Cover Change». En D.C. PARKER et al. (eds.): International Workshop. Irvine, California, USA.

PLATA ROCHA, W., GOMEZ DELGADO, M. y BOSQUE SENDRA, J. (2009): «Análisis de factores explicativos del crecimiento urbano en la $\mathrm{CM}$ a través de métodos estadísticos (RLO y MLA) y SIG». Revista de Planeamiento Territorial y Urbanismo Iberoamericana, 4.

PLATA ROCHA, W., GÓMEZ DELGADO, M. y BOSQUE SENDRA, J. (2010): «Desarrollo de modelos de crecimiento urbano óptimo para la CM aplicando Métodos de Evaluación Multicriterio y Sistemas de Información Geográfica». Geofocus, n 10, 103-134.

PLATA ROCHA, W., GÓMEZ DELGADO, M. y BOSQUE SENDRA, J. (2011): «Simulation urban growth scenarios using GIS and Multicriteria Evaluation techniques. Case study: Madrid Region, Spain». Environment and Planning B, $\mathrm{n}^{\circ}$ 38, 1012-1031.

RAILSBACK, S.F. y GRIMM, V. (2009): A Course in Individual-based and Agent-based Modeling, Princeton University Press.

SANDERS, L., PUMAIN, D., MATHIAN, H., GHERIN-PACE, F. y BURA, S. (1997): «SIMPOP: a multi-agents system for the study of urbanism». Environment and Planning $B, \mathrm{n}^{\circ} 24,287-305$.

SANTÉ, L., GARCÍA, A.M., MIRANDA, D. y CRESCENTE, R. (2010): «Cellular automata models for the simulation of real world urban processes: A review and analysis». Landscape and Urban Planning, $\mathrm{n}^{\circ}$ 96, 108-122.

SANTOS PRECIADO, J.M., AZÁRATE LUXÁN, M.V., MATESANZ, D.C., GARCÍA LÁZARO, F.J. y MUGURUZA CAÑAS, C. (2012): «Claves del crecimiento urbano 
actual». En M. GÓMEZ DELGADO y V.M. RODRÍGUEZ ESPINOSA (eds.): Análisis de la Dinámica Urbana y Simulación de Escenarios de Desarrollo Futuro con Tecnologías de la Información Geográfica. Madrid, RA-MA.

SERRANO CAMBRONERO, M.M., GAGO GARCÍA, C. y ANTÓN BURGOS, F.J. (2002): Impacto territorial de las carreteras orbitales de la Comunidad de Madrid. Madrid, Comunidad de Madrid.

SHELLING, T.C. (1971): «Dynamic Models of Segregation». Journal of Mathematical Sociology, $\mathrm{n}^{\circ} 1(2), 143-189$.

SIU: Sistema de Información Urbana. Disponible en http://visorsiu.fomento.es/siu/PortalSiu.html (acceso en Julio 2014).

TORRENS, P.M. (2006): «Geosimulation and its Application to Urban Growth Modeling». En J. PORTUGALI (ed.): Complex Artificial Environments (págs. 119-134). London, Springer-Verlag.

TORRENS, P.M. (2012): «Moving agent-pedestrians through space and time». Annals of the Association of American Geographers, $\mathrm{n}^{\circ}$ 102(1), 35-66.

VAN DELDEN, H., DÍAZ-PACHECO, J., SHI, I. y VAN VLIET, J. (2012): «Calibration of cellular autómata based land use models: lessons learnt from practical experience». En N.N. PINTO et al. (eds.): (págs. 295-297). Oporto, Department of Civil Engineering, University of Coimbra.

WHITE, R., ENGELEN, G. y ULJEE, I. (1997): «The use of constrained cellular automata for high resolution modeling of urban land use dynamics». Environment and Planning B, $n^{\circ} 24,323-343$. 
